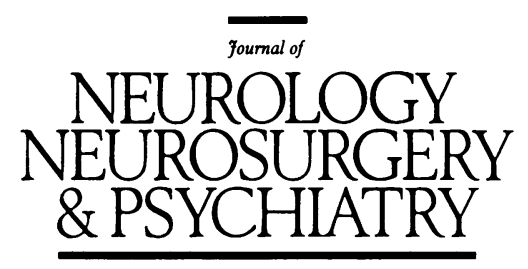

Editorial

\title{
The relevance to psychiatry of recent advances in functional imaging ${ }^{\star}$
}

The advent of functional imaging has revolutionised our capacity to understand both normal and abnormal brain function. Of the currently available techniques, positron emission tomography (PET) is the most informative because it allows accurate quantification of cerebral blood flow and metabolism and superior spatial resolution. As a direct consequence of the introduction of PET methodology a decade ago, significant advances have been made in our knowledge of conditions which have a recognised neuropathology. These include degenerative brain disease, cerebrovascular disease, movement disorders and epilepsy. Such studies have yielded important findings which are not only academically interesting but also clinically relevant. ${ }^{1}$ Perhaps the next great challenge for PET is the unravelling of the various neurobiological determinants of the so-called functional psychoses, schizophrenia and affective illness, since these are being increasingly recognised as having a substantial organic component. The early PET studies of these two conditions have provided some useful pointers towards the fundamental neuronal disturbances, but often the results from various PET centres have been inconsistent. For example some studies have found reduced activity in the frontal cortex of both depressed and schizophrenic patients whereas others have failed to replicate this finding. $^{2-4}$

There are several reasons for these discrepant results. First, neither schizophrenia nor affective illness is a unitary disorder. Both can be manifest by a variety of clinical features which presumably reflect the differential involvement of various neuronal systems. Thus variability with respect to clinical populations is one source of discrepancy. Second, the frontal cortex is not a unitary neuronal system being comprised of prefrontal association cortex, premotor and motor cortex, and cortex with neuroanatomical affiliation to the limbic system. Since the most commonly used method of image analysis is to place regions of interest, either by hand or by automated means, upon visually displayed two dimensional images, observer bias and inaccuracies of localisation also undoubtedly contribute to the variability of results. Recently, new automated methods of image analysis have been developed which compute group averaged images, subtract the common activity between groups and display an image representing statistically significant group differences in stereotactic space. ${ }^{56}$ These methods remove observer bias, provide more accurate localisation of function, and represent an important advance for PET research.
Advances have also been made in the experimental approach to PET. The ability to image the brain during different physiological states allows the application of paradigms derived from cognitive psychology. Cognitive psychology has provided us with detailed accounts of the component processes underlying human behaviour. Until now the neurobiological substrate of these processes have been inferred from human lesion studies but, because these are naturally occurring and therefore often crude, localisation cannot be precise. Animal research involving stereotactically placed lesions and single unit recordings, being more specific, is an alternative strategy. However, this has limitations. For example, animals cannot be used to investigate the basis of voluntary behaviour, because they cannot be asked to carry out acts which are truly voluntary. ${ }^{7}$ The ability to localise those neuronal systems involved in the performance of various mental operations is now possible by employing PET in healthy human beings. Furthermore, the use of the radioligand ${ }^{15} \mathrm{O}$ with a short half life provides the means to investigate several tasks in the same individual within the same experimental session, and thus obviates confounding problems of repositioning of the head and the need to ensure consistent ambient conditions necessary when tests are performed on different occasions. Finally, the development of sophisticated analytical techniques allows subtraction of images acquired under different test conditions. The activity found during control and experimental tasks can be subtracted to eliminate the activation common to both conditions as a result of, for example, sensory processing and thus those areas of the brain involved in higher mental function can be highlighted.

How can PET research into human cognition advance our knowledge of psychosis? For many years abnormal mental phenomena, the clinical signs of psychiatric disorder, have been conceptualised as reflecting various aberrant psychological processes. ${ }^{8-13}$ Although the phenomena exhibited by psychiatric patients are many and apparently diverse, they can be grouped into core syndromes $^{1415}$ each of which can be related to the disruption of a fundamental cognitive process. PET has allowed such theories to be tested because the regional activation underlying specific cognitive operations in normal volunteers can be directly compared with those regional abnormalities disclosed on the PET scans of psychiatric populations. In this way inferences can be made concerning the meaning of abnormal mental phenomena. This approach has been pioneered by workers at The Hammersmith Hospital MRC Cyclotron Unit, the 
results of which have either recently been published or communicated at the winter meeting of the British Neuropsychiaty Association (BNPA).

For example, one influential theory proposed by Frith ${ }^{11}$ argues that schizophrenic phenomena are secondary to a disturbance of the perception and initiation of action. He distinguishes between actions (movements, thoughts) which are elicited by external stimuli and those which are internally generated by intentions of will. Frith provides evidence to suggest that the positive features of schizophrenia, such as hallucinations and delusions, are secondary to the failure of a monitoring system which normally provides the conscious registration that an act has been willed. It is argued that negative features, such as poverty of speech and movement and flatness of affect, are due to the inability to generate intentions of will per se. Frith suggests that the neural substrate concerned with the formation of willed intentions lies within the prefrontal cortex, a notion compatible with Shallice's model which invokes the frontal cortex in planning ability. ${ }^{16}$ To test this hypothesis Frith et al $^{1718}$ have used PET to study regional cerebral blood flow (rCBF) in normal volunteers during the performance of routine and novel tasks in two modalities (word generation and finger movement). The two tasks differed in that the novel condition required the generation of volitional or willed responses whereas the routine condition required responses determined by the experimenter. When rCBF maps were compared, novel responses generated a common pattern of cerebral activation for both modalities which was not present during routine responses. Regional cerebral blood flow was increased in the dorsolateral prefrontal cortex, on the left in the word generation task and bilaterally in the finger movement task. They suggest that this area is "associated with the requirements to generate responses by willed action". A second region was also activated during the performance of willed acts, namely the anterior cingulate cortex which is situated on the medial aspect of the frontal lobe and which is considered limbic in nature. Mesulam ${ }^{19}$ has previously argued that this area of the brain plays a role in assigning motivational significance to sensory events so that meaningful stimuli can acquire greater impact than irrelevant stimuli for the direction of action. The anterior cingulate is therefore involved in "attention for action". 20 Frith et al propose that the anterior cingulate activation in their study represents this psychological process. The results of Frith's study can be related to a series of experiments in normal volunteers performed at the Washington University PET centre. Petersen $e t a l^{21}$ found increased rCBF in dorsolateral prefrontal cortex and anterior cingulate in a word generation task whereas Pardo et $a l^{22}$ found only cingulate activation during the performance of the Stroop task. Briefly, the Stroop task requires "attention for action" but not the generation of willed acts. Thus these results are compatible with the hypothesis that the dorsolateral prefrontal cortex and the anterior cingulate cortex subserve distinct cognitive processes both of which are required for the production of voluntary behaviour.

A different but related study of voluntary action was reported by Passingham ${ }^{7}$ in which normal volunteers were scanned while moving a joystick in one of four directions, at will, following a tone. This was compared with cerebral responses when the tone was presented alone or when the tone required a specific fixed response. During free selection they found increased activity in the dorsolateral prefrontal cortex, the anterior cingulate cortex and the adjacent supplementary motor cortex. On the basis of animal work, Passingham ${ }^{23}$ proposed that the supplementary motor cortex is involved in the learned selection of movements. Furthermore Posner et $a l^{20}$ noted that when motor output was involved in their tasks, medial frontal activity was located in the supplementary motor area whereas when attention to significant stimuli without the need for a motor response was involved, activity was located in the adjacent anterior cingulate cortex. Passingham proposes that a functionally integrated system involving dorsolateral prefrontal cortex, cingulate cortex and supplementary area, which can be influenced by motivation via connections with the amygdala and ventral striatum, subserves the performance of self initiated movements. In support of this contention Passingham reported a second study, involving the same paradigm, in patients with Parkinson's disease. A fundamental aspect of the movement disorder in Parkinson's disease is the inability to generate volitional movements since it is well recognised that these patients are much quicker to act when given external stimulation (for example, kinesia paradoxica). He found that patients with Parkinson's disease showed less activity in all of these three areas during the free selection condition but similar activity to normal volunteers during the control conditions.

Some discrepant results suggest that the relative involvement of dorsolateral prefrontal cortex, anterior cingulate and supplementary motor areas in the generation of voluntary behaviour require further clarification. For example, in Frith's study of voluntary action which involved motor behaviour, increased CBF within the supplementary motor area was not noted. Another discrepant result emerged from a study by Posner $^{20}$ which involved counting the number of dangerous animals in a list of visually presented words. This task activated the dorsolateral prefrontal cortex as well as the anterior cingulate region. As this task did not require the generation of intentions of will, it is difficult to explain this activation of dorsolateral cortex purely in terms of Frith's model.

Returning to the hypothesis which relates schizophrenic phenomena to an impairment in the perception and initiation of willed action, Frith $e t a l^{18}$ have used PET to investigate brain areas associated with self-monitoring in normal volunteers. They employed an ingenious eye movement paradigm which required the patient to monitor intended acts in order to produce the correct response. In the experimental phase, signals from an electroculogram were used to drive a stimulus on a monitor so that when the subject moved his eyes to the left, the stimulus moved to the right. Using eye movements, the subject was required to move the stimulus on the monitor, from side to side at a specified rate. To do this it was therefore necessary to correct for the distorted visual feedback by monitoring intended eye movements. Tasks were also employed to control for cerebral activity elicited by the visual feedback alone or eye movements alone. They found that the monitoring of intended movements was particularly associated with activity in the left hippocampus.

We can now turn from the results of these PET studies of normal volunteers to their relevance to an understanding of psychosis. In a previous series of studies on the categorisation of schizophrenic phenomena, Liddle 1524 $^{15}$ found three clusters or syndromes namely: psychomotor poverty (poverty of speech, flatness of affect and decreased spontaneous movement); disorganisation (disorders of the form of thought ) and reality distortion (hallucinations and delusions). Although these three syndromes are not mutually exclusive in any individual, they were proposed to represent abnormalities of distinct neuronal systems because each syndrome was found to be associated with different profiles of performance on neuropsychological tests. ${ }^{25}$ At the British Neuropsychiaty Association Winter Meeting 1992, Liddle reported that the features of the 
psychomotor poverty syndrome are primarily related to reduced resting cerebral blood flow in the left dorsolateral prefrontal cortex and anterior cingulate cortex. ${ }^{27}$ This therefore supports Frith's hypothesis ${ }^{11}$ that the negative features of schizophrenia are secondary to the inability to generate willed actions. Reality distortion was associated with increased activity in the left parahippocampal gyrus and can thus be related to Frith's hypothesis that positive schizophrenic phenomena are due to impaired monitoring of willed acts, a process localised to the left hippocampus. The frontal abnormality of CBF associated with the disorganisation syndrome was reduced activity in the right ventral prefrontal cortex and increased activity in the right anterior cingulate cortex. Although the neural basis of thought disorder has not been addressed by Frith, Liddle has found that this syndrome is associated with the inability to suppress inappropriate responses. ${ }^{26}$ Compatible with Liddle's findings, is the observation that lesions of the ventral prefrontal cortex in primates produce behavioural disinhibition. ${ }^{28}$ Liddle has suggested that the activation of the anterior cingulate cortex in patients with the disorganisation syndrome represents the ineffective struggle to suppress inappropriate mental activity. An alternative interpretation, with respect to the theory of anterior cingulate function described above, is that motivational significance is being wrongly ascribed to irrelevant stimuli in patients with thought disorder.

Arguably, certain features of depression may represent an impairment in the generation of willed acts. These are generally considered under the rubric of psychomotor retardation and include subjective feelings of loss of energy and objective signs of slowness and apathy. Dolan reported a study of depression in which 40 patients and 40 matched normal volunteers had resting CBF scans. ${ }^{2930}$ The depressed group as a whole demonstrated reduced frontal activity in the left anterior cingulate cortex and left dorsolateral prefrontal cortex. Correlational analysis revealed that psychomotor retardation was related to decreased $\mathrm{CBF}$ in the dorsolateral prefrontal cortex. Thus in depression as well as schizophrenia an impairment of the initiation of voluntary behaviour is found in association with decreased function of the dorsolateral prefrontal cortex, supporting the notion that this finding is due to failure of a fundamental psychological function rather than an attribute of a specific disease process.

Although there are many issues that remain to be examined, the findings of distinct associations between mental phenomena, cognitive acts and regional brain abnormalities is remarkable. We can therefore look forward to a decade in which the growing body of knowledge concerning the genetic, neurochemical and neuropathological basis of various psychiatric disorders will be related to abnormal psychological function using PET.

Although this article has concentrated on the implications of advances in PET for psychiatric research a second method of functional imaging which is as yet in its infancy should be briefly mentioned. This is proton spectroscopy using MRI. Gadian described at the British Neuropsychiaty Association's Winter Meeting how MRI provides a noninvasive method of studying brain metabolism in vivo. He pointed out that although initial MRI studies investigated energy metabolism by examining ${ }^{31} \mathrm{P}$ signals generated from ATP, phosphocreatine and inorganic phosphate moieties, the newly developed ${ }^{1} \mathrm{H}$ spectroscopy may be of more interest. It offers better spatial resolution than the ${ }^{31} \mathrm{P}$ technique and it can measure proton signals from neurophysiologically important chemicals such as $\mathbf{N}$-acetylaspartate (NAA) and glutamate. For example, NAA is found almost exclusively in neurons and therefore is a putative marker of neuronal loss or damage. Glutamate, an excitatory neurotransmitter, has been implicated as a neurotoxic agent in various disorders including schizophrenia. ${ }^{31}$ It is likely that MRI spectroscopy will be a complementary rather than an alternative technique to PET. PET is able to detect dysfunctional brain areas but cannot distinguish, whether these are secondary to a cellular abnormality in the areas directly under inspection or the effects of remote lesions disrupting the integrity of projection systems to the site of the detected abnormalities. Furthermore, a recent structural MRI study reported by Ron, ${ }^{32}$ has demonstrated that schizophrenic patients have reduced global cerebral volume, a finding seemingly at odds with the PET data which suggests that the functional abnormalities are localised to specific brain areas. The presence of global brain atrophy does not imply global brain dysfunction. MRI spectroscopy has the ability to detect the consequences of neuronal damage for cellular metabolism in various regions and may provide a bridge between findings derived from MRI structural imaging and PET functional imaging of the brain in schizophrenia and other psychiatric disorders.

EILEEN M JOYCE Academic Department of Psychiatry Charing Cross and Westminster Medical School, London

«This was the theme of the The British Neuropsychiatry Association Winter Meeting, London, January 1992

1 Brooks DJ. PET: its clinical role in neurology. $\mathcal{F}$ Neurol Neurosurg Psychiatry 1991;54:1-4

2 Buchsbaum M, DeLisi L, Holcomb H, et al. Anteroposterior gradients in cerebral glucose use in schizophrenia and affective disorders. Arch Gen Psychiat 1984;41:1159-66.

3 Shepherd G, Manchanda R, Gruzelier, et al. $\mathrm{O} 15$ positron emission tomography scanning in predominantly never-treated acute schizophrenic patients. Lancet 1983;2:1448-52.

4 Joyce EM. Cerebral blood flow and metabolism in affective disorders. Int $\mathfrak{J}$ Geriatric Psychiat 1991;6:423-30.

5 Fox PT, Mintum MA, Reiman EM, Raichle ME. Enhanced detection of focal brain responses using intersubject PET images. 7 Cereb Blood Flow Metab 1988;8:642-53.

6 Friston KJ, Frith CD, Kiddle PF, et al. Comparing functional (PET) images: the assessment of significant change. $\mathcal{F}$ Cereb Blood Flow Metab 1991; 11:690-9.

7 Deiber M-P, Passingham RE, Colebatch JG, Friston KJ, Nixon PD, Frackowiak RSJ. Cortical areas and the selection of movement: a study with positron emission tomography. Exp Brain Res 1991;84:393-402.

8 Broen WE, Storms LH. A theory of response interference in schizophrenia. In: Maher, ed. Progress in experimental personality reseach, Vol 4. New York: Academic Press, 1967:269-312.

9 McGhie A, Chapman J. Disorders of attention and perception in early schizophrenia. Brit $\mathcal{F}$ Med Psychol 1961;34:103-116.

10 Hemsley DR. An experimental psychological model for schizophrenia. In Hafner H, Gattaz WF, Janzavik W, eds. Search for the cause of schizophrenia. Heidelberg: Springer-Verlag, 1987:170-88.

11 Frith $C D$. The positive and negative symptoms of schizophrenia reflect impairments in the perception and intitiation of action. Psychol Med 1987;7:631-8.

12 Robbins TW. Cognitive deficits in schizophrenia and Parkinson's disease: Neural basis and the role of dopamine. In: Willner $P$, Scheel-Krugeer J, Neural basis and the role of dopamine. In: Willner P, Scheel-Krugeer eds. The Mesolimbic dopamine

13 Robbins TW, Sahakian BJ. Behavioural effects of psychomotor stimulant drugs: clinical and neuropsychological implications. In: Creese I, ed. Stimulants: neurochemical, behavioural and clinical perspectives. New York Raven Press. 1983:301-8.

14 Crow TJ. Molecular pathology of schizophrenia: more than one disease process? $B M \mathcal{F}$ 1980;280:66-8.

15 Liddle PF. The symptoms of chronic schizophrenia. Brit $f$ Psychiat 1987a;151:145-51.

16 Shallice T. Specific impairments in planning. Phil Trans Roy Soc Med Lond 1982;298:199-209.

17 Frith CD, Friston KJ, Liddle PF, Frackowiak RSJ. Willed action and the prefrontal cortex in man: a study with PET. Proc Roy Soc Lond prefrontal corte

18 Frith CD, Friston KJ, Liddle PF, Frackowiak RSJ. PET imaging and cognition in schizophrenia. $\mathcal{F}$ Roy Soc Med 1992;85:222-4.

S Mesulam M-M. A cortical network for directed attention and unilateral neglect. Ann Neurol 1981;10:309-25.

0 Posner MI, Petersen SE, Fox PT, Raichle ME. Localisation of cognitive operations in the human brain. Science 1988;240:1627-31

1 Petersen SE, Fox PT, Posner MI, Mintum M, Raichle ME. Positron emission studies of the cortical anatomy of single-word processing. Nature 1988;331:585-9.

22 Pardo JV, Pardo PJ, Janer KW, Raichle ME. The anterior cingulate cortex mediates processing selection in the Stroop attentional conflict paradigm. Proc Natl Acad Sci USA 1990;87:256-9.

23 Passingham RE. Premotor cortex and preparation for movement. Exp Brain Res 1988;70:590-6. 
24 Liddle PF, Barnes TRE. Syndromes of chronic schizophrenia. Brit $\mathfrak{f}$ Psychiat 1990;157:558-61.

25 Liddle PF. Schizophrenic syndromes, cognitive performance and neurological dysfunction. Psychol Med 1987b;17:49-57.

26 Liddle PF, Morris DL. Schizophrenic syndromes and frontal lobe performance. Brit f Psychiat 1991;158:140-345.

27 Liddle PF, Friston KJ, Frith CD, Hirsch SR, Jones T, Frackowiak RSJ. Patterns of cerebral blood flow in schizophrenia. Brit $\mathcal{f}$ Psychia 1992;160:179-86.

28 Fuster JM. The prefrontal cortex. New York: Raven Press 1980

29 Bench CJ, Scott LC, Brown RG, Friston KJ, Frackowiak RSJ, Dolan RJ.
Regional cerebral blood flow in depression determined by positron emission tomography. 7 Cereb Blood Flow Metab 1991;11(Suppl 2):654

30 Dolan RJ, Bench CJ, Scott LC, Brown RG, Friston KJ, Frackowiak RSJ. Regional cerebral blood flow in 'depressive pseudodementia' determined by positron emission tomography. $\mathcal{F}$ Cereb Blood Flow Metab 1991;11 (Suppl 2):S17

31 Royston MC, Simpson MDC. Post-mortem neurochemistry of schizophrenia. In: Kerwin R, ed. Neurobiology and psychiatry, Vol 1. Cambridge: Cambridge University Press, 1991:1-14.

32 Ron MA, Harvey I, Lewis SW, Murray RM. Diffuse structural brain abnormalities in schizophrenia. Schizophrenia Res 1992;6:145.

\section{Neurological stamp}

\section{Jean Nicot 1530-1600}

When he was French ambassador to Lisbon Jean Nicot was intrigued by local reports of the efficacy of tobacco for the cure of some serious diseases. On his return from Portugual he introduced the tobacco plant into France (hence the name nicotine). It was used to cure headaches, treat syphilis, and the smoke was thought to alleviate asthma. The green leaves were advised for neck pain.

It is said that tobacco cured the queen of her headaches and that those in positions of influence experimented with the plant and promoted the therapeutic aspects associated with it. Tobacco became a fixed part of European medical practice. It was prescribed for a multitude of ailments and diseases including paralysis, rabies, tetanus, epilepsy, nervous disorders and hysteria.

There was a popular suspicion at the time that doctors opposed the smoking of tobacco because it was diminishing the need for their services. In later years there was a general attack upon the use of tobacco. Horace Greeley, an American journalist and politician defined the cigar as "a fire at one end and a fool at the other."

The 400th anniversary of the introduction of tobacco into France was marked by a stamp issued in 1961 (Stanley Gibbons no 1517, Scott no 989).

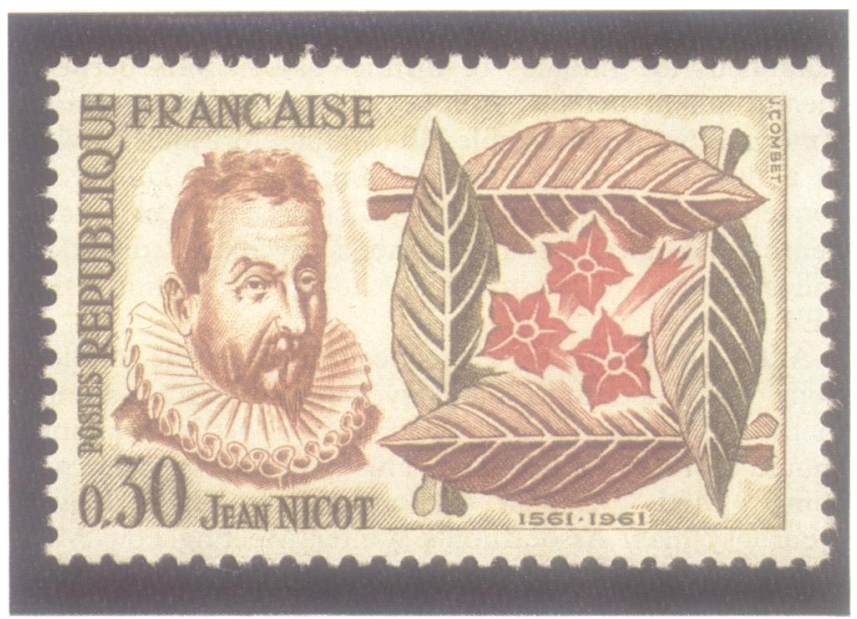

